\title{
Hydrogen atoms in circularly polarized microwave fields: Near-integrability and ionization
}

\author{
M. J. Raković and Shih-I Chu \\ Kansas Institute for Theoretical and Computational Science and Department of Chemistry, \\ University of Kansas, Lawrence, Kansas 66045
}

(Received 14 March 1995)

\begin{abstract}
We have recently found that the hydrogen atom in a circularly polarized (CP) microwave field possesses an approximate dynamical symmetry and its bounded motion can be well described by a three-dimensional integrable (but nonseparable) Hamiltonian function with a velocity-dependent potential [Raković and Chu, Phys. Rev. A 50, 5077 (1994)]. This finding provides a theoretical foundation for the understanding of the origin of the regularity of Rydberg atom dynamics in CP fields. We describe here the phase space topology of the three-dimensional integrable system relevant to the microwave ionization of the hydrogen atoms in CP fields. Using the integrable system as an approximation to the real system and with the use of the two additional integrals of motion, we are able to trace the deformation of the tori up to the point of bifurcation (ionization). From this, we have determined the classical ionization-field threshold law $f_{\text {th }} \approx 1 / c n_{0}^{4}$, where $n_{0}$ is the principal quantum number of the initial state of the hydrogen atom and $c$ is almost a constant $(\approx 6 \mathrm{a} . \mathrm{u}$.). These results are in good accord with the existing experimental observations.
\end{abstract}

PACS number(s): 32.80.Rm, 03.20. $+\mathrm{i}, 32.60 .+\mathrm{i}$

\section{INTRODUCTION}

In the past decade considerable attention has been paid to the study of the ionization of Rydberg atoms in linearly polarized (LP) microwave fields $[1,2]$. This is a nonintegable system that exhibits classically chaotic motion and has served as one of the paradigms for the study of "quantum chaos." A related problem, of more recent interest, is the ionization of Rydberg atoms in the presence of circularly polarized (CP) microwave fields. A recent experiment [3] on microwave ionization of Rydberg (sodium) atoms has revealed a dramatic difference of the behavior in LP and CP fields. First, it was observed that the ionization threshold fields $f_{\text {th }}$ in CP fields are considerably higher than those in the LP fields. Further, the ionization thresholds in the CP fields obey the scaling law characteristic for the case of the classical hydrogen atom in the static field (Stark effect), i.e., $f_{\text {th }} \approx 1 / c n_{0}^{4}$, where $n_{0}$ is the principal quantum number of the hydrogenic initial state and $c$ is almost a constant. Several recent theoretical studies $[4,5]$ of the restricted motion in the plane of the polarization of the CP field have shown that the classical motion is almost completely regular below the classical ionization threshold. This regularity of the Rydberg atom motion in $\mathrm{CP}$ fields is unexpected as the classical dynamics of the corresponding Rydberg systems in the LP fields can be "very chaotic."

The motivation of this paper is twofold. First, we shall theoretically analyze the origin of the regularity of motion in CP fields. Second, we shall study the classical ionization threshold law for the case of hydrogen atom in CP microwave fields, providing a prediction for the constant $c$. In connection with this, we note that microwave ionization of atomic hydrogen in CP fields is currently being pursued experimentally. Our prediction of the ionization threshold law is found to be in excellent agree- ment with the preliminary experimental results [6].

We shall study the ionization of the Rydberg states of the hydrogen atom in circularly polarized microwave fields from the standpoint of classical mechanics. Recall that the Hamiltonian function (atomic units will be used throughout this paper unless otherwise indicated) of the hydrogen atom in circularly polarized field $H_{\text {cir }}=\mathrm{p}^{2} / 2-1 / r+f(x \cos \omega t+y \sin \omega t)$ has, in the frame rotating around the $z$ axis together with the field $(x \rightarrow x \cos \omega t-y \sin \omega t, y \rightarrow x \sin \omega t+y \cos \omega t)$, the timeindependent form

$$
H_{\mathrm{cir}}=\frac{\mathrm{p}^{2}}{2}-\frac{1}{r}-\omega l_{z}+f x,
$$

where $\mathbf{p}=\left(p_{x}, p_{y}, p_{z}\right), l_{z}=x p_{y}-y p_{x}$, and $f$ and $\omega$ are, respectively, the amplitude and the frequency of the applied microwave field. Essential for our treatment is the fact that, although this system is not integrable, almost all bounded trajectories are regular for all intensities of the applied field provided that the frequency is small enough. This regularity is the consequence of the approximate dynamical symmetry of the system, i.e., of its nearintegrability $[5,7]$.

In a previous paper [7] we have discovered that the dynamical system with the Hamiltonian function

$$
H=\left(\frac{\mathrm{p}^{2}}{2}-\frac{1}{r}-\omega l_{z}+f x\right)+\frac{\omega^{2}}{18}\left(r^{2}+3 z^{2}\right)
$$

is completely integrable. Let us name the systems corresponding to the Hamiltonian functions (1) and (2) as systems I and II, respectively. Note that the global topological structure of the phase spaces corresponding to two systems is quite different. System II allows only bounded trajectories and since it is integrable, almost all its trajectories are contained in the invariant three-dimensional 
tori in the phase space. On the other hand, system I supports both bounded and unbounded (scattering, ionizing) trajectories. However, since

$$
V=H-H_{\mathrm{cir}}=\frac{\omega^{2}}{18}\left(r^{2}+3 z^{2}\right),
$$

one might expect that for small enough $\omega$, e.g., for the microwave fields that are of current interest in this study, the bounded trajectories of system I, located in the vicinity of the nucleus, can be well approximated by the (regular) trajectories of the integrable system II. This explains the regularity of system $I$ in the region of the phase space that contains bounded trajectories. The relation between the two systems will be explored in detail in Sec. III. Before that, we shall describe in Sec. II the topological structure of the phase space corresponding to the integrable system II. Finally, in Sec. IV, within the framework of the adiabatic theory, we shall use the integrable system II as a zeroth-order approximation to calculate ionization threshold fields for the hydrogen atom in circularly polarized microwave field.

\section{PHASE-SPACE STRUCTURE OF THE INTEGRABLE SYSTEM II}

Recall that an $n$-dimensional Hamiltonian system is integrable if it possesses $n$ independent integrals of motion that are in involution. In the case of the threedimensional integrable system II two additional [besides the Hamiltonian function itself, Eq. (2)] integrals of motion are [7]

$$
\begin{aligned}
I= & \frac{4}{3} \omega l_{z}(H-f x)+\frac{8}{9} \omega^{2} l_{z}^{2}+f\left(l_{y} p_{z}-l_{z} p_{y}+\frac{x}{r}\right) \\
& +\frac{f^{2}}{2}\left(y^{2}+z^{2}\right)+\frac{1}{3} \omega f\left(3 x l_{z}-p_{y} r^{2}+z l_{x}\right) \\
& -\frac{1}{9} \omega^{2} f x\left(r^{2}+z^{2}\right), \\
J= & \omega\left(l_{x} p_{y}-l_{y} p_{x}+\frac{z}{r}\right) \\
& +\frac{1}{9} \omega^{3} z\left(x^{2}+y^{2}\right)-\frac{3}{2} f l_{x}-\frac{1}{2} \omega f x z .
\end{aligned}
$$

Both systems I and II possess the following useful scaling property: if one introduces the scaled canonical coordinates $\mathbf{r}_{s}=\omega^{2 / 3} \mathbf{r}$ and $\mathbf{p}_{s}=\omega^{-1 / 3} \mathbf{p}$ and the scaled field intensity $f_{s}=\omega^{-4 / 3} f$, then the scaled Hamiltonian functions $H_{\text {cir }}^{s}=\omega^{-2 / 3} H_{\text {cir }}$ and $H_{s}=\omega^{-2 / 3} H$ and scaled integrals of motion $I_{s}=\omega^{-4 / 3} I$ and $J_{s}=\omega^{-1} J$ have the same functional forms as given in Eqs. (1)-(5) with the replacement $\omega=1$. Further, both systems I and II possess a discrete symmetry. The Hamiltonian functions $H_{\text {cir }}$ and $H$, as well as the function $I$, are invariant under the transformation $\left(x, y, z, p_{x}, p_{y}, p_{z}\right) \rightarrow(x, y,-z$, $\left.p_{x}, p_{y},-p_{z}\right)$, while the function $J$ transforms as $J \rightarrow-J$.

\section{A. Invariant sets of the integrable system II}

Let $P$ be a phase space of both systems I and II. Consider the energy-momentum map [8] corresponding to the integrable system II:

$$
\begin{aligned}
& M_{\mathrm{EM}}: P \rightarrow \mathbb{R}^{3}:(\mathbf{r}, \mathbf{p}) \rightarrow(H(\mathbf{r}, \mathbf{p}), I(\mathbf{r}, \mathbf{p}), J(\mathbf{r}, \mathbf{p})), \\
& \mathcal{M}_{\mathrm{EM}}(P)=Q .
\end{aligned}
$$

where $Q \subset \mathbb{R}^{3}$ is the image of the phase space $P$ under the energy-momentum map. Let $\boldsymbol{M}(\mathbf{r}, \mathbf{p})$ be the differential of the energy-momentum map, i.e., the $(3 \times 6)$ Jacobian matrix,

$$
M(\mathbf{r}, \mathbf{p})=\left(\begin{array}{ll}
\partial H / \partial \mathbf{r} & \partial H / \partial \mathbf{p} \\
\partial I / \partial \mathbf{r} & \partial I / \partial \mathbf{p} \\
\partial J / \partial \mathbf{r} & \partial J / \partial \mathbf{p}
\end{array}\right)
$$

The phase space naturally decomposes into a (disjoint) union of the level sets of the energy-momentum map (i.e., inverse images of the points from $Q$ )

$$
\begin{aligned}
\mu_{E, C_{1}, C_{2}} & =\left\{(\mathbf{r}, \mathbf{p}): H(\mathbf{r}, \mathbf{p})=E, I(\mathbf{r}, \mathbf{p})=C_{1}, J(\mathbf{r}, \mathbf{p})=C_{2}\right\} \\
& =M_{\mathrm{EM}}^{-1}\left(E, C_{1}, C_{2}\right), \quad\left(E, C_{1}, C_{2}\right) \in Q .
\end{aligned}
$$

Each level set $\mu_{E, C_{1}, C_{2}}$ is invariant under the motion corresponding to the Hamiltonian function $H$, as well as under the motions of $I$ and $J$ treated as Hamiltonian functions. The topological structure of the given level set depends on whether the corresponding point from $Q$ is regular or not. Recall [8] that the point $\left(E, C_{1}, C_{2}\right) \in Q$ is regular if it is a regular value of the energy-momentum map $\mathcal{M}_{\mathrm{EM}}$ or, in other words, if for each $(\mathbf{r}, \mathbf{p}) \in \mu_{E, C_{1}, C_{2}}$ one has that $\operatorname{rank}[M(\mathbf{r}, \mathbf{p})]=3$; otherwise the point ( $E, C_{1}, C_{2}$ ) is said to be critical. In other words, if the point from $Q$ is regular, then at each phase-space point belonging to the corresponding level set the three Hamiltonian vector fields generated by the functions $H, I, J$ are linearly independent. Let $Q_{r}$ and $Q_{c}$ be the sets of all regular and singular points, respectively. It is easy to verify that rank $[M(\mathbf{r}, \mathbf{p})]$ is invariant under the discrete transformation $\quad\left(x, y, z, p_{x}, p_{y}, p_{z}\right) \rightarrow\left(x, y,-z, p_{x}, p_{y},-p_{z}\right)$. Therefore the sets $Q, Q_{r}$, and $Q_{c}$ are all invariant under the transformation $\left(E, C_{1}, C_{2}\right) \rightarrow\left(E, C_{1},-C_{2}\right)$. According to Liouville's theorem [8], if the point $\left(E, C_{1}, C_{2}\right)$ is regular then the invariant level set $\mu_{E, C_{1}, C_{2}}$ is a threedimensional smooth manifold. In particular if $\mu_{E, C_{1}, C_{2}}$ is compact and connected, then it is diffeomorphic to a three-dimensional torus. Since the integrable system II allows only bounded motions, the level sets of regular points are necessarily compact and some of them are connected. As we shall soon see, if such a level set is not connected, then it is diffeomorphic to a disjoint union of exactly two three-dimensional tori. On the other hand, if the point $\left(E, C_{1}, C_{2}\right)$ is critical, then the corresponding level set necessarily contains (as subsets) invariant smooth manifolds of dimension lower than three, which if compact and connected, are diffeomorphic to twodimensional tori, circles (periodic orbits), or (fixed) points.

We shall now describe the topological structure of the sets of regular and singular points corresponding to system II. They are both subsets of $\mathbb{R}^{3}$, i.e., $Q_{r} \cup Q_{c}=Q \subset \mathbb{R}^{3}$. The set $Q_{r}$ is three-dimensional open 
(in $\mathbb{R}^{3}$ ) and disconnected set, while $Q_{c}$ decomposes into several subsets of different dimensions. The convenient way to represent these sets is to give plots of intersections of the set $Q$ with various planes of the space $\mathbb{R}^{3}=\left\{\left(E, C_{1}, C_{2}\right)\right\}$ defined by fixing the third integral $C_{2}$. (From now on in this subsection we shall use the scaled variables $f_{s}=\omega^{-4 / 3} f, E_{s}=\omega^{-2 / 3} E, C_{1 s}=\omega^{-4 / 3} C_{1}$, and $C_{2 s}=\omega^{-1} C_{2}$.) Such intersections belong to (for a given value of the scaled parameter $f_{s}$ ) four different topological classes. The first class consists of only one element: it is the intersection of $Q$ with the plane $C_{2 s}=0$. The second class of topologically equivalent sets contains intersections of $Q$ with planes for which $0<\left|C_{2 s}\right|<1$, while the third and the fourth classes contain intersections of $Q$ with the planes for which $1<\left|C_{2 s}\right|<C_{2 G}$ [where $C_{2 G}=C_{2 G}\left(f_{s}\right)$; see the Appendix] and $\left|C_{2 s}\right|>C_{2 G}$, respectively. We note here that the intersection of $Q$ with the plane $C_{2 s}=0$ can have two different structures depending on whether $f_{s}>\left(\frac{4}{9}\right)^{2 / 3}$ or $f_{s} \leq\left(\frac{4}{9}\right)^{2 / 3}$, while the topological structure of the intersection of $Q$ with the planes $C_{2 s} \neq 0$ does not depend on $f_{s}$. In Figs. 1(a)-1(d) we have given (for $f_{s}=2.67$ ) four plots each representing one class. We see that the set $Q_{r}$ consists of two open connected components $Q_{r}=Q_{1} \cup Q_{2}$. The set of critical points $Q_{c}$ contains four two-dimensional subsetssurfaces $\sigma_{1}, \sigma_{2}, \epsilon_{1}$, and $\epsilon_{2}$-and four one-dimensional subsets-curves $l_{1}, l_{2}, l_{3}$, and $l_{4}$. The points belonging to shaded areas in Figs. 1(a)-1(d) are not elements of the set $Q$, i.e., the union of the critical surfaces $\sigma_{1}$ and $\epsilon_{2}$ is the boundary of the closed (in $\mathbb{R}^{3}$ ) set $Q$. In Fig. 1(a) we see that the three curves $l_{1}, l_{2}$, and $l_{3}$ lie entirely in the $C_{2 s}=0$ plane. The lines $l_{2}$ and $l_{3}$ exist only if $f_{s}>\left(\frac{4}{9}\right)^{2 / 3}$, i.e., the triangle $A F_{2} B$ contracts as $f_{s}$ decrease and for $f_{s}=\left(\frac{4}{9}\right)^{2 / 3}$ it reduces to a point. From Fig. 1(b) and 1(c) we see that the open regular set $Q_{1}$ has nonempty intersections only with the planes for which $0 \leq\left|C_{2 s}\right|<C_{2 G}$ and these intersections are bounded for $1<\left|C_{2 s}\right|<C_{2 G}$, Fig. 1(c). Also we see that the curve $l_{4}$ intersects only the planes for which $0 \leq\left|C_{2 s}\right|<1$, Fig. 1(b). This curve is also represented in Figs. 2(a) $-2(\mathrm{c})$. Note that the curves $l_{2}, l_{3}$, and $l_{4}$ meet at the point $B$. Note that the regular set $Q_{1}$ is simply connected (and diffeomorphic to $\mathbb{R}^{3}$ ) while, due to location of the critical curves $l_{1-4}$, the set $Q_{2}$ is not simply connected. Analytical formulas for all surfaces and curves belonging to the set $Q_{c}$ are summarized in the Appendix.
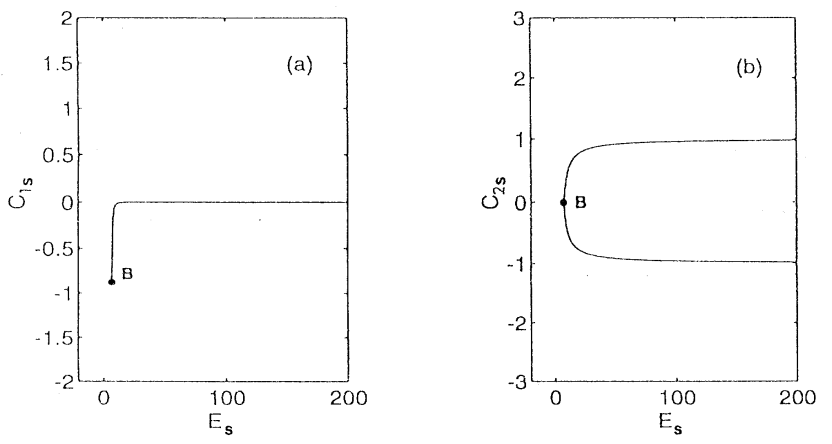
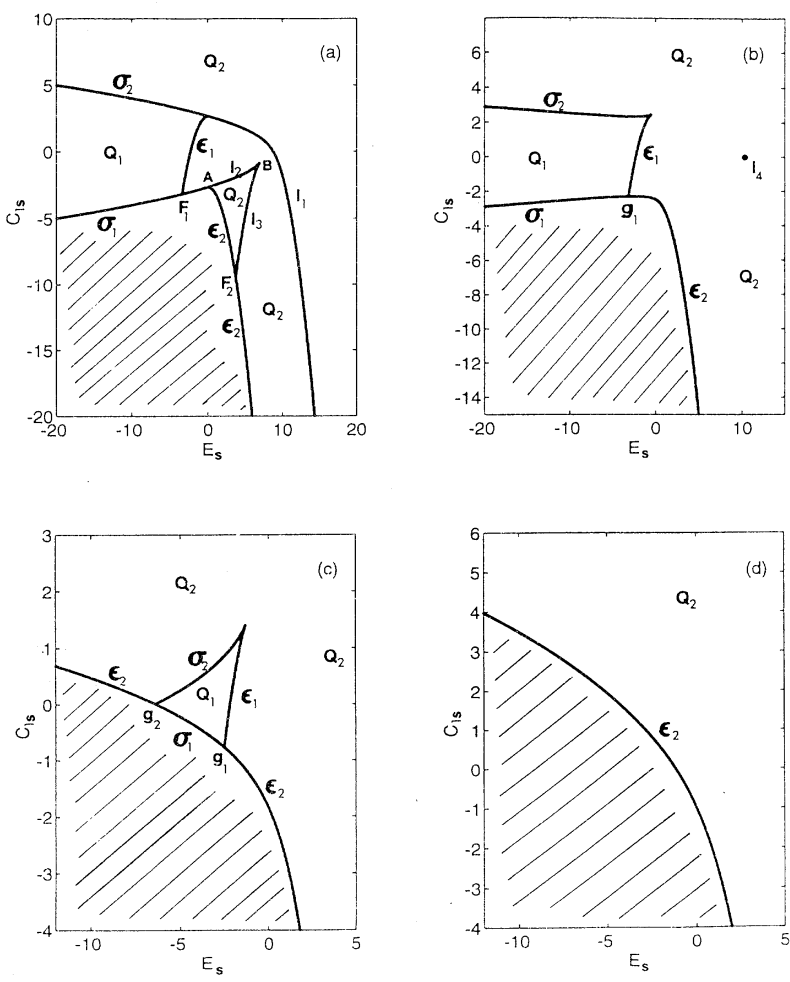

FIG. 1. Intersections of the set $Q$ (see the text) and the planes for which (a) $\quad C_{2 s}=0, \quad$ (b) $\left|C_{2 s}\right|=0.5, \quad$ (c) $\left|C_{2 s}\right|=1.5<C_{2 G}=2.352$, and (d) $\left|C_{2 s}\right|=2.5>C_{2 G}$. The scaled field intensity is $f_{s}=2.67$. All quantities are given in atomic units.

Consider now the level sets Eq. (8) corresponding to regular points belonging to the set $Q_{2}$. Each such level set is connected and thus diffeomorphic to the threedimensional torus. Therefore the set $Q_{2}$ corresponds to a three-parameter family of three-dimensional tori, which we shall denote with $w_{2}$. In Figs. 1(a)-1(d) we see that the union of the critical surfaces $\sigma_{2}, \epsilon_{1}$, and $\varepsilon_{2}$ is the boundary of the closure of the open set $Q_{2}$. One may think that the family of tori $w_{2}$ cannot be extended across that boundary of critical points and this is indeed true for the surfaces $\epsilon_{1}$ and $\epsilon_{2}$. The level sets corresponding to the critical points from these surfaces are also connected, but they are not diffeomorphic to three-dimensional tori. The level sets of the points from $\epsilon_{2}$ are diffeomorphic to two-dimensional tori and can be viewed as if they are ob-

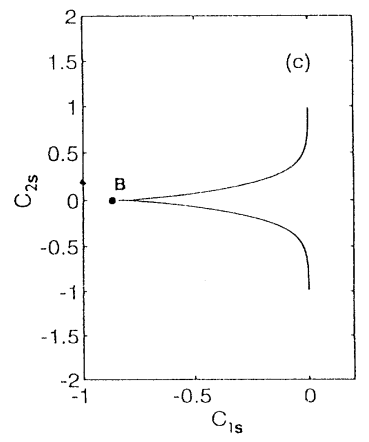

FIG. 2. Plot of the critical curve $l_{4}$. (a) Projection onto the $C_{1 s}-C_{2 s}$ plane. (b) Projection onto the $E_{s}-C_{2 s}$ plane. (c) Projection onto the $C_{1 s}-C_{2 s}$ plane. The scaled field intensity is $f_{s}=2.67$. All quantities are given in atomic units. 
tained by the contraction of the tori from the family $w_{2}$. The level sets of the points $\epsilon_{1}$ have more complicated structure (see below). However, the family $w_{2}$ can be extended across the critical surface $\sigma_{2}$ into the open regular set $Q_{1}$. This is because the level set of any given point from $\sigma_{2}$ is not connected: it is a disjoint union of one three-dimensional torus, naturally embedded in the family $w_{2}$, and one two-dimensional torus. Consistent with that is the fact the level set corresponding to any regular point from $Q_{1}$ is also not connected: it is a disjoint union of two three-dimensional tori, one of which belongs to the family $w_{2}$ and another to the (new) family $w_{1}$. Clearly, the two-dimensional tori that correspond to the points from $\sigma_{2}$ are obtained by the contraction of the threedimensional tori of the family $w_{1}$. Neither family of tori can be extended across the critical surface $\sigma_{1}$. The level sets of the points from that surface consist of two disjoint two-dimensional tori. The most interesting is the critical surface $\epsilon_{1}$. The connected level sets of the points from that surface are the so-called separatrices: they separate two families of tori. Each such level set decomposes into three invariant subsets: one unstable [under the motion generated by the Hamiltonian function $H$, Eq. (2)] twodimensional torus and two noncompact threedimensional sets (diffeomorphic to direct products of two circles and a real line), which constitute unstable and stable manifolds of the unstable two-dimensional torus. (Note that invariant two-dimensional tori that correspond to the critical points from the surfaces $\sigma_{1}, \sigma_{2}$, and $\epsilon_{2}$ are all stable.)

In the conclusion of this subsection we define sets $W_{1}$ and $W_{2}$ to be the unions of the tori belonging to families $w_{1}$ and $w_{2}$, respectively. By construction they are two six-dimensional open disjoint subsets of the phase space foliated by the corresponding three-parameter families of invariant three-dimensional tori. In the next subsection we shall use the technique of surfaces of section to illustrate some of the level sets described above. A more detailed analysis of the topological structure of the level sets, especially of those corresponding to the critical points and various bifurcation phenomena, will be addressed elsewhere [9].

\section{B. Reduced two-dimensional systems}

For dynamical systems, with more degrees of freedom than one, the standard method for graphical presentation of invariant sets (trajectories) is the method of Poincare surfaces of section in which one constructs intersection of the given invariant set with some suitable transversal subset (with the codimension one) of the phase space. In the case of our integrable system II the most suitable subset for the construction of the surfaces of section (SOS) is the five-dimensional plane $z=0$ because it is transversal to all invariant sets $\mu_{E, C_{1}, C_{2}}$ with $C_{2} \neq 0$. To verify that, first not that the Hamiltonian vector field $X_{H}=(\partial H / \partial \mathrm{p},-\partial H / \partial \mathrm{r})$ of the Hamiltonian function $H$, Eq. (2), is tangent to all level sets $\mu_{E, C_{1}, C_{2}}$. On the other hand, the vector $\mathbf{n}=(0,0,1,0,0,0)$ is orthogonal to the plane $z=0$. Therefore, for the plane $z=0$ to be transver-

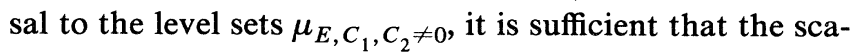
lar product $\mathrm{n} \cdot X_{H}=\partial H / \partial p_{z}=p_{z}$ is different from zero at the phase-space points for which $z=0$ and $C_{2} \neq 0$. But from Eq. (5) we see that if $J=C_{2} \neq 0$ and $z=0$, then $p_{z} \neq 0$. From this consideration we also see that the intersection of the plane $z=0$ and the level set $\mu_{E, C_{1}, C_{2} \neq 0}$ consists of two disjoint subsets that belong to two fivedimensional half planes defined with the conditions $p_{z}>0$ and $p_{z}<0$.

Moreover, to each $C_{2} \neq 0$ corresponds an integrable two-dimensional system $\mathrm{II}_{C_{2}}$ such that the intersection of the plane $z=0$ with any level set $\mu_{E, C_{1}, C_{2}}$ for which $J=C_{2}$ is related to an invariant set of the system $\mathrm{II}_{C_{2}}$. The Hamiltonian function $H_{C_{2}}\left(x, y, p_{x}, p_{y}\right)$ and additional integral of motion $I_{C_{2}}\left(x, y, p_{x}, p_{y}\right)$ of the system $\mathrm{II}_{C_{2}}$ are obtained from the Eqs. (2) and (4) by setting $z=0$ and replacing $p_{z}$ with the solution (for $p_{z}$ ) of the equation $\left.J\right|_{z=0}=C_{2}$,

$$
\begin{aligned}
H_{C_{2}}= & \frac{p_{x}^{2}+p_{y}^{2}+p_{z 0}^{2}}{2}-\frac{1}{\left(x^{2}+y^{2}\right)^{1 / 2}} \\
& -\omega l_{z}+f x+\frac{1}{18} \omega^{2}\left(x^{2}+y^{2}\right), \\
I_{C_{2}}= & \frac{4}{3} \omega l_{z}\left(H_{C_{2}}-f x\right)+\frac{8}{9} \omega^{2} l_{z}^{2} \\
& +f\left[-p_{z 0}^{2} x-p_{y} l_{z}+\frac{x}{\left(x^{2}+y^{2}\right)^{1 / 2}}\right] \\
& +\frac{1}{2} f^{2} y^{2}+\frac{1}{3} \omega f\left[3 x l_{z}-p_{y}\left(x^{2}+y^{2}\right)\right] \\
& -\frac{1}{9} \omega^{2} f x\left(x^{2}+y^{2}\right),
\end{aligned}
$$

where

$$
p_{z 0}=\frac{C_{2}}{\omega\left(p_{y} y+p_{x} x\right)-3 f y / 2} .
$$

Now, given some invariant three-dimensional torus (from the family $w_{1}$ or $w_{2}$ ) with the corresponding regular point $\left(E, C_{1}, C_{2}\right) \in Q_{r}$, its intersection with the plane $z=0$ consists of two disjoint two-dimensional tori (which lie in the half planes $p_{z}>0$ and $p_{z}<0$ ). The projections of these two-dimensional tori on the (four-dimensional) space $\mathbb{R}^{4}=\left\{\left(x, y, z=0, p_{x}, p_{y}, p_{z}=0\right)\right\}$ are invariant sets of the system $\mathrm{II}_{C_{2}}$ and are subsets of the level set defined with the conditions $H_{C_{2}}=E$ and $I_{C_{2}}=C_{1}$.

As for the case of $C_{2}=0$, it appears that the plane $z=0$ is not necessarily transversal to the level sets $\mu_{E, C_{1}, C_{2}=0}$. However, it is easy to verify that the four-dimensional subset of the phase space, defined with $z=0$ and $p_{z}=0$ and for which $C_{2}=0$ [see Eq. (5)], is invariant under the motion of the Hamiltonian function $H$, Eq. (2), as well as under the motion generated by the function $I$, Eq. (4), treated as a Hamiltonian function. Therefore, the restriction of system II to the plane $z=0, p_{z}=0$ defines an integrable two-dimensional system $\mathrm{II}_{0}$ with the following Hamiltonian function and additional integral of motion: 


$$
\begin{aligned}
H_{0}= & \frac{p_{x}^{2}+p_{y}^{2}}{2}-\frac{1}{\left(x^{2}+y^{2}\right)^{1 / 2}} \\
& -\omega l_{z}+f x+\frac{1}{18} \omega^{2}\left(x^{2}+y^{2}\right), \\
I_{0}= & \frac{4}{3} \omega l_{z}\left(H_{0}-f x\right)+\frac{8}{9} \omega^{2} l_{z}^{2} \\
& +f\left[-l_{z} p_{y}+\frac{x}{\left(x^{2}+y^{2}\right)^{1 / 2}}\right]+\frac{1}{2} f^{2} y^{2} \\
& +\frac{1}{3} \omega f\left[3 x l_{z}-p_{y}\left(x^{2}+y^{2}\right)\right] \\
& -\frac{1}{9} \omega^{2} f x\left(x^{2}+y^{2}\right) .
\end{aligned}
$$

Now, given some invariant three-dimensional torus (from the family $w_{1}$ or $w_{2}$ ) with the corresponding regular point $\left(E, C_{1}, 0\right)$, its intersection with the plane $z=0$, $p_{z}=0$ consists of (one or two) two-dimensional tori that are invariant sets of system $\mathbf{I I}_{0}$.

In Figs. 3 and 4 we show the plots of several level sets of the two-dimensional system $\mathrm{II}_{0}$ (in the case $f_{s}=2.67$ ) corresponding to various regular and critical points. The
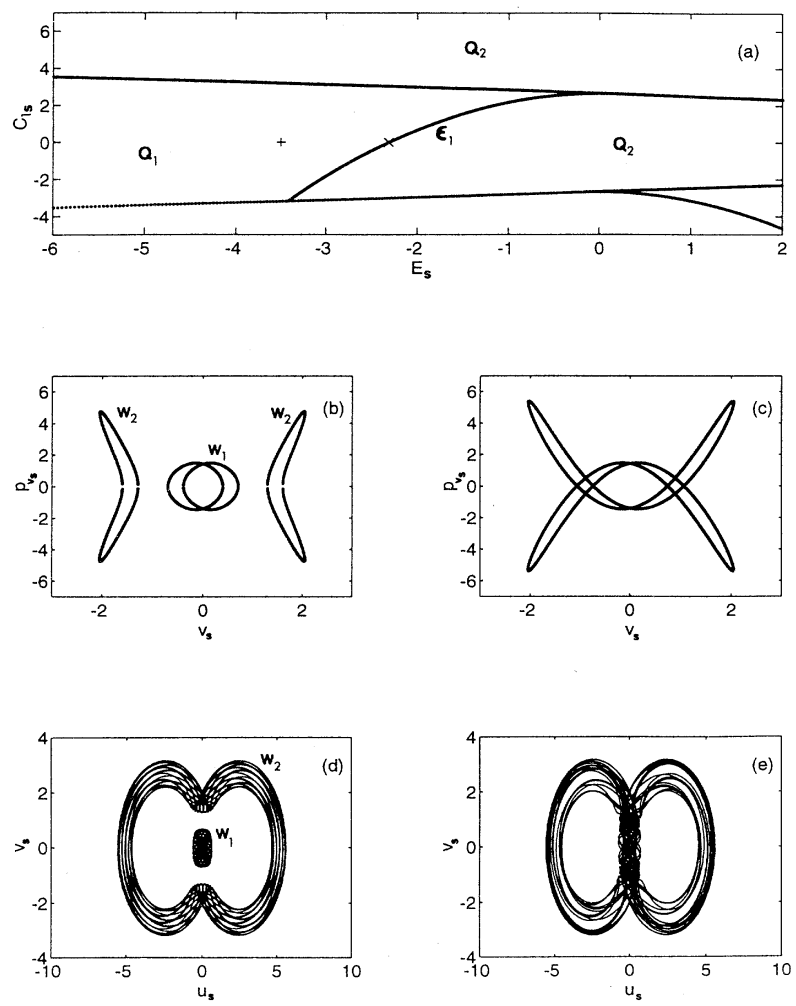

FIG. 3. Invariant level sets of a regular point from the set $Q_{1}$ and a critical point from the surface $\epsilon_{1}$ corresponding to twodimensional system $\mathrm{II}_{0}$. The scaled field intensity is $f_{s}=2.67$. (a) Positions of the regular and the critical points. (b) SOS, defined with $u=0$, of the level set corresponding to the regular point. (c) SOS of the level set corresponding to the critical point. (d) Projection onto the $u-v$ plane, of two tori $w_{1}$ and $w_{2}$, corresponding to the regular point. (e) Projection, onto the $u-v$ plane, of the sepatrix corresponding to the critical point. All quantities are given in atomic units.
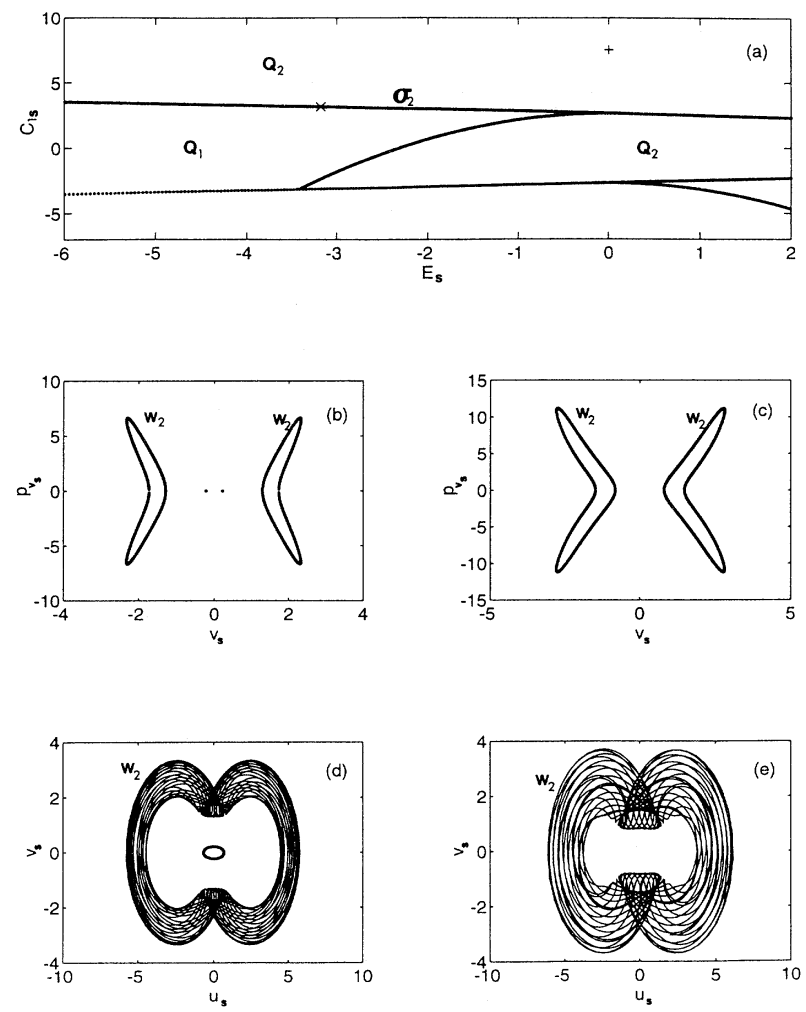

FIG. 4. Same as in Fig. 3 but for (b) and (d) the critical point from the surface $\sigma_{2}$ and (c) and (e) the regular point from $Q_{2}$.

semiparabolic coordinates, which regularize the Coulomb singularity, are defined with the equations

$$
\begin{aligned}
& x=\left(u^{2}-v^{2}\right) / 2, \quad y=u v, \\
& p_{x}=\left(u p_{u}-v p_{v}\right) /\left(u^{2}+v^{2}\right), \\
& p_{y}=\left(v p_{u}+u p_{v}\right) /\left(u^{2}+v^{2}\right)
\end{aligned}
$$

and in Figs. 3 and 4 we have used the corresponding scaled variables $u_{s}=\omega^{1 / 3} u, v_{s}=\omega^{1 / 3} v, p_{u_{s}}=p_{u}$, and $p_{v_{s}}=p_{v}$. Shown in Fig. 3(a) are the locations of a regular point from the set $Q_{1}$ and critical points from the surface $\epsilon_{1}$. The corresponding level sets are represented in Figs. 3(b)-3(e). The SOS's are defined as the intersections of the given level sets and the plane $u=0$. In the case of the regular point, Fig. 3(b), it consists of four disjoint closed curves (diffeomorphic to circles) whose projections on the $v-p_{v}$ plane are shown in Fig. 3(b). One pair of these "circles" belongs to a two-dimensional invariant torus of system $\mathrm{II}_{0}$, which is the intersecting set of the corresponding three-dimensional invariant torus from the family $w_{1}$ of system II with the plane $z=0, p_{z}=0$. Analogously the other pair of the circles belongs to the torus from the family $w_{2}$. Projections of these two-dimensional tori onto the configurational plane $u-v$ are given in Fig. 3(d). (In fact we plot the trajectories of the motion generated by the Hamiltonian function $H_{0}$ obtained by evolving randomly chosen initial point, one from each torus.) The level set of the critical point from the surface $\epsilon_{1}$ is presented 
in Figs. 3(c) and 3(e). We see that two tori from the families $w_{1}$ and $w_{2}$ "meet" at this point and the corresponding level set of system $\mathbf{I I}_{0}$, the separatrix, consists of one invariant one-dimensional set (unstable periodic orbit) and two invariant two-dimensional noncompact sets, both diffeomorphic to cylinders. These cylinders constitute stable and unstable manifolds of the unstable (periodic) orbit. Shown in Fig. 4(a) are the locations of a critical point from the surface $\sigma_{2}$ and a regular point from the set $Q_{2}$. The corresponding level sets of system $\mathrm{II}_{0}$ are represented in Figs. 4(b)-4(e). In the case of the critical point, Figs. 4(b) and 4(d), the level set consists of one two-dimensional torus (the intersection of the corresponding torus from the family $w_{2}$ with the plane $z=0$, $p_{z}=0$ ) and, as expected (the critical point), one onedimensional orbit. The level set of the regular point from the set $Q_{2}$, Figs. 4(c) and 4(e), is a single two-dimensional torus (the intersection of the corresponding torus from the family $w_{2}$ with the plane $\left.z=0, p_{z}=0\right)$. Figures $5(\mathrm{~b})-5(\mathrm{~d})$ show the SOS's of the level sets of the twodimensional system $\mathrm{II}_{C_{2}}$ (for $C_{2 s}=1.1$ and $f_{s}=2.67$ ) that correspond to three regular points from $Q_{1}$, while Fig. 5(e) shows the SOS corresponding to the singular point from the surface $\epsilon_{1}$. In this case we have used polar coor-
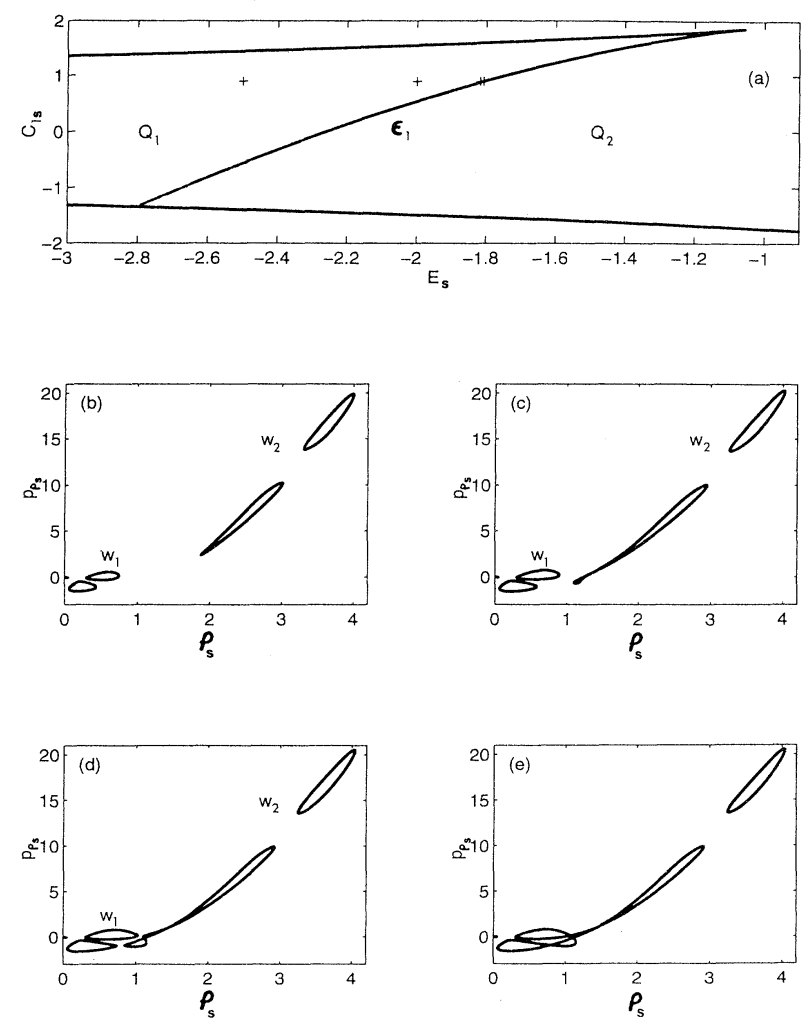

FIG. 5. SOS's of the invariant level sets of the system $\mathbf{I I}_{C_{2}}$ corresponding to (b)-(d) three regular points from the set $Q_{1}$ and (e) a critical point from the surface $\epsilon_{1}$. The location of the points are given in (a). The parameters are $f_{s}=2.67$ and $C_{2 s}=1.1 \mathrm{SOS}$ is defined with $\phi=0.3 \pi$. All quantities are given in atomic units. dinates in $u-v$ plane, i.e., after the introduction of the semiparabolic coordinates, Eq. (13), we define the following set of canonically conjugate coordinates:

$u=\rho \cos \phi, \quad v=\rho \sin \phi$,

$p_{u}=p_{\rho} \cos \phi-\frac{p_{\phi}}{\rho} \sin \phi, \quad p_{v}=p_{\rho} \sin \phi+\frac{p_{\phi}}{\rho} \cos \phi$.

Then the SOS is defined by fixing the polar coordinate $\phi$. Again, we have used the scaled variables $\rho_{s}=\omega^{1 / 3} \rho$, $p_{\rho_{s}}=p_{\rho}$, and $p_{\phi_{s}}=\omega^{1 / 3} p_{\phi}$.

We conclude this subsection by commenting on the difference between invariant tori of system II depending on whether $C_{2} \neq 0$ or $C_{2}=0$. As is well known [8], in the case of the integrable (nondegenerate) Hamiltonian systems almost all bounded trajectories are everywhere dense in the invariant tori to which they belong. It is said that the motion in the invariant torus is ergodic. In the case of our system II this is true for almost all invariant tori (from the families $w_{1}$ and $w_{2}$ ) for which $C_{2} \neq 0$. On the other hand, the trajectories for which $C_{2}=0$ are not everywhere dense in the corresponding tori. Instead, each invariant three-dimensional torus with $C_{2}=0$ decomposes into three invariant subsets and trajectories of $H$ are everywhere dense only in these subsets. One of these subsets is a two-dimensional torus-an invariant set of the integrable system $\mathrm{II}_{0}$-i.e., it is the intersection of the given three-dimensional torus with the plane $z=0$, $p_{z}=0$. The other two subsets are three dimensional and diffeomorphic to the direct products of two circles and a real line.

\section{Actions corresponding to invariant tori}

The invariant tori (one or two; see Sec. II A) that correspond to a given regular point $\left(E, C_{1}, C_{2}\right) \in Q_{r}$ are implicitly defined by Eqs. (2), (4), and (5) with the conditions $H=E, \quad I=C_{1}$, and $J=C_{2}$. Three action variables $S_{1}, S_{2}, S_{3}$ corresponding to a given invariant threedimensional torus are defined [8] as integrals (divided by $2 \pi$ ) of the canonical one-form $\mathbf{p} d \mathbf{r}$ over three independent noncontractible loops belonging to the torus. Since there are infinitely many ways to choose threeindependent noncontractible loops for a given torus, the actions are not uniquely defined: the triplet $S=\left(S_{1}, S_{2}, S_{3}\right)$ is defined only up to the multiplication with any matrix $N$ with integer elements for which $|\operatorname{det}(N)|=1$, i.e., $S^{\prime}=N S$ is also a triplet of action variables associated with the same torus [10]. Consider now an open neighborhood $U$ of some regular point belonging to $Q_{1}$ such that $U \subset Q_{1}$. Then [8] there exist two (differentiable) maps associated with two families of tori $w_{1}$ and $w_{2}$

$\chi_{i}: U \rightarrow \mathbb{R}^{3}:\left(E, C_{1}, C_{2}\right) \rightarrow\left(S_{1}^{i}, S_{2}^{i}, S_{3}^{i}\right), \quad i=1,2$,

such that $S_{1}^{i}, S_{2}^{i}, S_{3}^{i}$ are the action variables of the torus that belongs to the family $w_{i}$ and corresponds to the regular point $\left(E, C_{1}, C_{2}\right)$. It is possible to extend continuously the function $\chi_{1}$ so that it is defined in the entire (open) set $Q_{1}$, i.e., one can define the "global" action 
coordinates that cover the family $w_{1}$ of three-dimensional tori. These actions, together with the corresponding canonically conjugate angle coordinates, form global action-angle coordinates [11] in the subset $W_{1}$ (see the last paragraph of Sec. II A) of the phase space. Again, due to nonuniqueness of the action variables, the set of (global) action coordinates that covers the family $w_{1}$ is defined up to the multiplication with the integer matrix with determinant \pm 1 . In order to cover the family $w_{2}$ of tori with the action coordinates one has to extend the function $\chi_{2}$ so that it is defined on $Q_{1} \cup \sigma_{2} \cup Q_{2}$ (recall that the level sets of the points from the critical surface $\sigma_{2}$ contain tori of the family $w_{2}$ ). However, due to the fact that the set $Q_{2}$ is not simply connected, such an extended function $\chi_{2}$ is multivalued (or it is not continuous if it is single valued) [11]. Therefore it is not possible to define global action coordinates for the family of tori $w_{2}$ or to introduce the global action-angle coordinates in the (open) subset $W_{2}$ of the phase space.

Let us now describe the technique we have used to calculate action variables of the tori from the family $w_{1}$, i.e., to construct (numerically) the function $\chi_{1}$. As we shall see in Sec. III only these tori are good approximations for the (existing) invariant tori of the realistic system $I$. The nontrivial part of this calculation is the location of three independent noncontractible loops $\gamma_{1}, \gamma_{2}$, and $\gamma_{3}$ belonging to the torus $T_{E, C_{1}}^{3}, C_{2}$ from the family $w_{1}$ defined with the values of integrals of motion $\left(E, C_{1}, C_{2}\right)$ and Eqs. (2), (4), and (5). As we know from Sec. II B, each such torus contains as a subset (at least one) the invariant twodimensional torus $T_{E, C_{1}}^{2}, C_{2}$ of the two-dimensional integrable system $\mathrm{II}_{C_{2}}$ defined with the values of the integrals of motion $\left(E, C_{1}\right)$ and Eqs. (9) and (10) for $C_{2} \neq 0$ or Eqs. (11) and (12) for $C_{2}=0$. The two independent loops, say, $\gamma_{1}$ and $\gamma_{2}$, can be chosen so that they belong to $T_{E, C_{1}, C_{2}}$. Therefore, the first step in the calculation is to locate one independent noncontractible loop $\gamma_{1}$ that belongs to $T_{E, C_{1}}^{2}, C_{2}$. This can always be done with the help of the suitable foliation of the four-dimensional phase space of the system $\mathrm{II}_{C_{2}}$ with the one-parameter family of the three-dimensional leaves. The foliation can always be chosen in such a way that almost all leaves are either transversal to the given torus (such leaves are obviously SOS) or do not intersect it at all. Transversal leaves (SOS) intersect the torus in one or a finite number of (disjoint) circles. Among those SOS's there always exist some SOS's such that at least one of their intersecting circles is noncontractible. That is our first noncontractible loop $\gamma_{1}$ and the corresponding action $S_{1}$ is the integral of the canonical one-form $(1 / 2 \pi)\left(p_{x} d x+p_{y} d y\right)$ along $\gamma_{1}$. In order to define the suitable foliation of the phase space of the system $\mathrm{II}_{C_{2}}$, we have introduced two canonical transformations (that have already been used for the definition of SOS's; see Sec. II B) generated by the changes of configurational coordinates $(x, y) \rightarrow(u, v) \rightarrow(\rho, \phi)$ given in Eqs. (13) and (14). The leaves of foliation are then defined as the level sets of coordinate $u$ when $C_{2}=0$ and the polar angle (in the $u-v$ plane) $\phi$ when $C_{2} \neq 0$. The SOS's in Figs. 3-5 contain noncontractible loops obtained in this way. Once we have located the first noncontractible loop and the corresponding SOS, the second independent noncontractible loop is obtained in the following way. In the loop $\gamma_{1}$ we choose some point $G_{1}$ as the initial point and integrate equations of motion corresponding to the Hamiltonian function $H_{C_{2}}$, until the trajectory intersects the circle $\gamma_{1}$ (i.e., crosses the corresponding SOS) at some point $G_{2}$. Connecting the points $G_{2}$ and $G_{1}$ with the arc belonging to $\gamma_{1}$ we obtain the second independent noncontractible loop $\gamma_{2}$ of the given invariant torus and again the second action is defined as the integral of the canonical one-form $(1 / 2 \pi)\left(p_{x} d x+p_{y} d y\right)$ along $\gamma_{2}$. The ambiguity in the choice of the arc that connects $G_{2}$ and $G_{1}$ leads to nonuniqueness of the action variables as explained above. Two values for the second action $S_{2}$ and $S_{2}^{\prime}$ corresponding to the two arcs connecting $G_{2}$ and $G_{1}$ differ exactly by the value of the first action $S_{1}$. The method for the location of the third loop is not the same for the cases $C_{2} \neq 0$ and $C_{2}=0$. Consider first the case $C_{2} \neq 0$. We again take some point $G_{1}$ from the loop $\gamma_{1}$ as the initial point, but now we first integrate equations of motion corresponding to Hamiltonian function $H$, Eq. (2) (of the full system II). At the beginning the trajectory leaves the torus $T_{E, C_{1}, C_{2}}^{2}$ and we integrate equations of motion until the trajectory returns and intersects the torus $T_{E, C_{1}, C_{2}}^{2}$ at some point $G_{3}$. Starting from that point, we again integrate equations of motion corresponding to the Hamiltonian function $H_{C_{2}}$, until the trajectory intersects the loop $\gamma_{1}$ at some point $G_{4}$. We then obtain the third loop $\gamma_{3}$ by connecting the points $G_{4}$ and $G_{1}$ with one of the two arcs that belong to the loop $\gamma_{1}$. The third action $S_{3}$ is obtained by the integration of the (full) one-form $(1 / 2 \pi) \mathbf{p} d \mathbf{r}$ along the loop $\gamma_{3}$. When $C_{2}=0$ the trajectories of the Hamiltonian function $H_{0}$ coincide with those of $H$ (see Sec. II B). In this case, in the construction of the loop $\gamma_{3}$, the initial trajectory (from $G_{1}$ to $G_{3}$ ), which leaves the torus $T_{E, C_{1}, C_{2}}^{2}$ and returns to it, is generated by the motion corresponding to the function $J$ treated as a Hamiltonian function.

\section{RELATION BETWEEN SYSTEMS I AND II}

The analysis of the topological structure of the phase space has shown that all trajectories of the Hamiltonian system II are bounded; almost all of them are contained in three-dimensional invariant tori that form two threeparameter families $w_{1}$ and $w_{2}$. Our main goal in this paper is to use system II as the approximation for the motion of system I, i.e., of the hydrogen atom in the circularly polarized (microwave) fields. As we mentioned in the Introduction, such an approach is justified only for those trajectories of system II for which the difference between two Hamiltonian functions, Eq. (3), is small. This is obviously not true for the trajectories that belong to the invariant tori of the family $w_{2}$, i.e., those that lie in the subset $W_{2}$ of the phase space. Indeed, the trajectories of system $I$ that lie in $W_{2}$ are unbounded, i.e., they corre- 
spond to scattering-ionizing motion. On the other hand, the trajectories belonging to tori from the family $w_{1}$ are located in the vicinity of the nucleus (see Sec. II) and for them, if $\omega$ is sufficiently low, the difference between two Hamiltonian functions is a small perturbation. In other words, in the region $W_{1}$ of the phase space and for low frequencies, system I can be viewed as near integrable, i.e., as the system obtained by a perturbation of the integrable system II. Therefore, according to the Kol'mogorov-Arnol'd-Moser (KAM) theorem [8], the majority of trajectories of system $I$ that lie in $W_{1}$ are regular, i.e., they are contained in the invariant tori that can be viewed as slightly deformed tori of the integrable system II. Only the small portion (proportional to the perturbation parameter, in this case $\omega^{2} / 18$ ) of the subset $W_{1}$ is filled by irregular trajectories not contained in invariant tori. For given parameters $f$ and $\omega$ we define $w_{\text {cir }}=w_{\text {cir }}(f, \omega)$ to be a collection of invariant tori from the family $w_{1}$ with the following property: if $T_{E, C_{1}, C_{2}}^{3} \in w_{\text {cir }}$ [then $\left.\left(E, C_{1}, C_{2}\right) \in Q_{1}\right]$ and if $\left(S_{1}, S_{2}, S_{3}\right)$ are the corresponding action variables, then there exists a nearby-lying invariant torus $T_{\text {cir }}^{3}$ of the (nonintegrable) system I that can be obtained by slight deformation of $T_{E, C_{1}}^{3}, C_{2}$ and that has the same values for the action variables. Let $Q_{\text {cir }}$ and $W_{\text {cir }}$ denote the corresponding subsets of $Q_{1}$ and $P$, respectively. According to the KAM theorem we have that $v\left(Q_{1} / Q_{\text {cir }}\right) / v\left(Q_{1}\right) \sim \omega^{2} / 18$, where $v$ is a measure defined on the set $Q$. An analogous relation holds for the sets $W_{\text {cir }}$ and $W_{1}$. In order to estimate the "size" of the set $Q_{\text {cir }}=Q_{\text {cir }}(f, \omega)$ we have to compare the SOS of systems I and II for various values of parameters $f$ and $\omega$. Due to the scaling property (see the introduction to Sec. III) we can set $\omega=1$ and vary only the scale parameter $f_{s}=f \omega^{-4 / 3}$. In Fig. 6 we show the SOS's (defined with $u=0$ and $\dot{u}>0$ ) that correspond to invariant tori of systems I [Figs. 6(d) and 6(e)] and II [Figs. 6(b) and 6(c)] for the motion in the plane of polarization $\left(z=0, p_{z}=0\right)$ for which $C_{2}=0$. The corresponding values of the scaled invariants $E_{s}$ and $C_{1 s}$ of system II are given in Fig. 6(a). The scaled parameter $f_{s}=2.67$ corresponds to the typical values of the ionization threshold field in the experiment of $\mathrm{Fu}$ et al. [3], i.e., $f_{s}=2.67 \rightarrow \omega=8.5 \mathrm{GHz}$ and $f=193 \mathrm{~V} / \mathrm{cm}$. The chosen scaled energies $E_{s}=-2$ [Fig. 6(b)] and $E_{s}=-1$ [Fig. 6(c)] formally correspond to the states of the unperturbed hydrogen atom with the principal quantum numbers $n \approx 46$ and 65 , respectively. In Figs. $6(\mathrm{~d})$ and $6(\mathrm{e})$ we see that (for these values of the parameters) the motion of system $I$ is almost completely regular and that the invariant tori of the two systems practically coincide. Also we see that almost all tori of the family $w_{1}$ (of system II ), for which $E_{s}=-2$ and $E_{s}=-1$, belong to the collection $w_{\text {cir }}$, with the exception of the tori located very close to the separatrix, i.e., whose corresponding points $\left(E_{s}, C_{1 s}, C_{2 s}=0\right)$ lie in the very small vicinity of the critical surface $\epsilon_{1}$ of the set $Q_{c}$. For these tori the corresponding motion of system $I$ is unbounded, i.e., ionizing motion. Similarly, Fig. 7 shows, for the case $f_{s}=1.35$, several SOS's of the two systems. We see that for higher
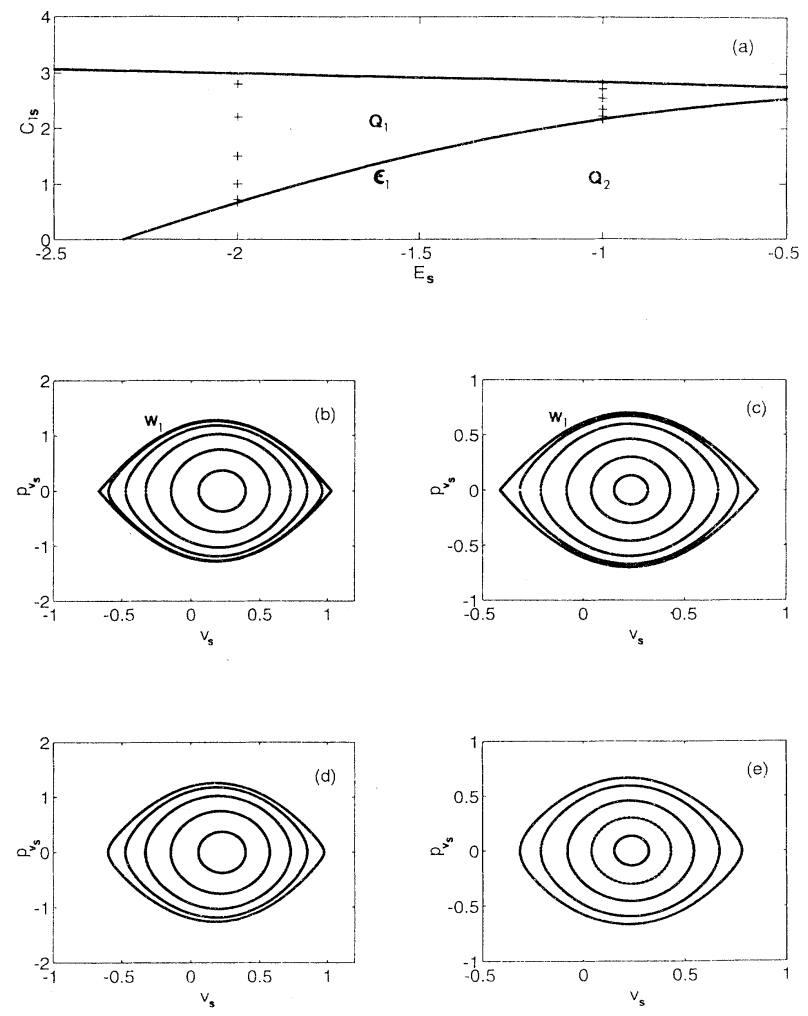

FIG. 6. SOS's, defined with $u=0$ and $\dot{u}>0$, that correspond to several invariant tori of (d) and (e) system $I$ and (b) and (c) system II for the motion in the plane of the polarization $(z=0$, $p_{z}=0$ ). The points from the set $Q$ that correspond to tori (family $w_{1}$ ) of system II are shown in (a). The scaled energies of the tori of the system II are (b) $E_{s}=-2$ and (c) $E_{s}=-1$. The scaled field intensity is $f_{s}=2.67$. All quantities are given in atomic units.

energies $E_{s}$, the vicinity of the separatrix filled by tori of family $w_{1}$ not belonging to $w_{\text {cir }}$ is larger [compare Figs. $7(\mathrm{c})$ and $7(\mathrm{e})$ ]. An extensive comparison of the SOS's for various values of parameters has shown that for all values of $f_{s}$ the set $Q_{\text {cir }}$ practically coincides with $Q_{1}$ except for the narrow strip near the critical surface $\epsilon_{1}$. Let the surface $\epsilon_{\text {cir }}$ be the boundary of the set $Q_{\text {cir }}$. Our empirical comparison of SOS's has revealed that for any $f_{s}$ the portion of $\epsilon_{\mathrm{cir}}$ corresponding to lower energies, i.e., $E_{s}<-1.5$ [or equivalently $\omega(-E)^{-3 / 2}<0.55$ ] practically coincides with $\epsilon_{1}$, so that for these energies the critical surface $\epsilon_{1}$ corresponds to the boundary between bounded (regular) and unbounded (ionizing) motion of system $\mathbf{I}$. For higher energies the surface $\epsilon_{\text {cir }}$ curves away from the surface $\epsilon_{1}$ and the volume of the subset $W_{1} \backslash W_{\text {cir }}$ of the phase space filled with irregular and/or unbounded trajectories of system I becomes larger.

\section{DETERMINATION OF IONIZATION THRESHOLD FIELDS: ADIABATIC APPROACH}

We treat ionization of the hydrogen atom by circularly polarized microwave field as an adiabatic process, i.e., we 

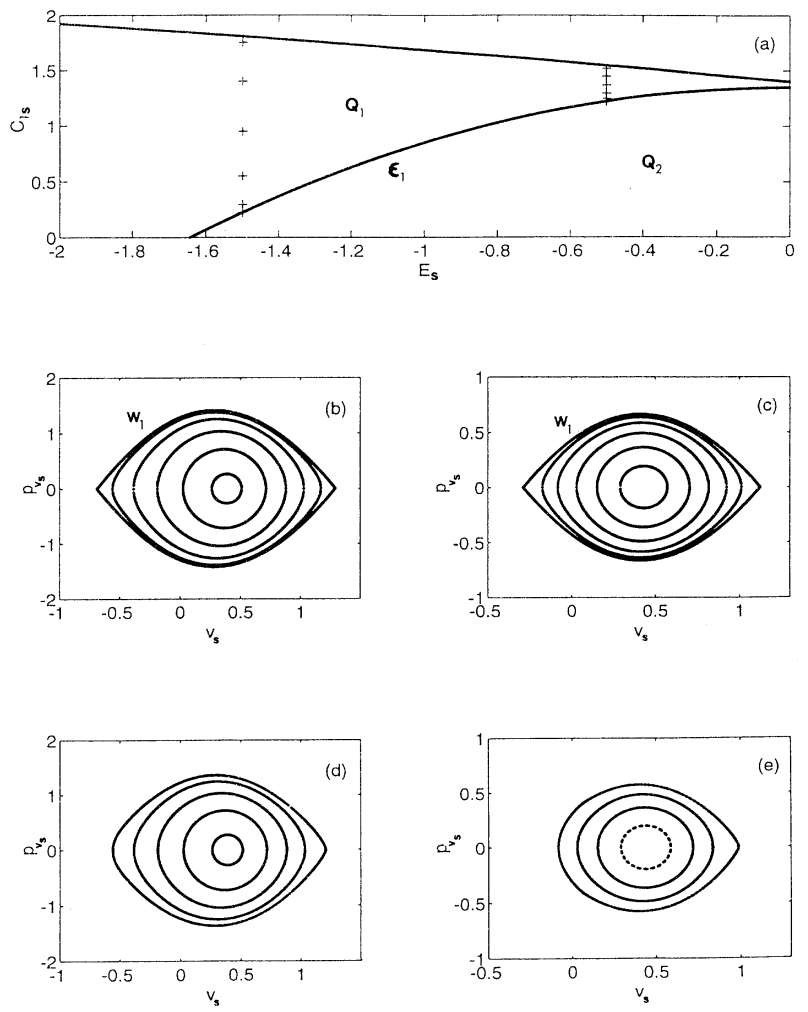

FIG. 7. Same as in Fig. 6 but for $f_{s}=1.35$ and (b) $E_{s}=-1.5$ and (c) $E_{s}=-0.5$.

assume that the hydrogen atom is initially prepared in the bound state with a given energy $E_{0}<0$ (or principal quantum number $n_{0}=1 / \sqrt{-2 E_{0}}$ ) and that the microwave field is slowly switched on. More precisely, the field intensity $f$ is turned on adiabatically from zero to some final value $f_{1}$ during the time period $T$, i.e., $f(0)=0, f(t)=f_{1}$ for $t \geq T$, and $0<\dot{f}(t) / f_{1} \ll \omega$ for $0 \leq t\langle T \gg 1 / \omega$. However, the field switching time $T$ is still much shorter then the quantum tunneling time scale. This is consistent with the experimental conditions. From the standpoint of classical mechanics the initial state is then described by the microcanonical ensemble of uniformly distributed phase-space points on the energy shell $E_{0}$ or, in other words, the probability distribution of the phase-space points $(\mathbf{r}, \mathbf{p})$ in the initial state is

$$
d w(\mathbf{r}, \mathbf{p})=\mathrm{const} \times \delta\left(E_{0}-H_{0}(\mathbf{r}, \mathbf{p})\right) d \mathbf{r} d \mathbf{p}, \quad \int d w=1,
$$

where $H_{0}$ is the Hamiltonian function of the unperturbed hydrogen atom. The hydrogen atom is a highly degenerate integrable dynamical system and all its trajectories are periodic. However, since in the rotating frame the Hamiltonian function of the unperturbed hydrogen atom takes the form [see Eq. (1)] $H_{0}-\omega l_{z}$, it is convenient to view the initial five-dimensional energy shell as foliated by a two-parameter family of invariant tori corresponding to, apart from the Hamiltonian function $H_{0}$, two additional integrals of motion: the $z$ projections of the angular momentum and the Runge-Lentz vector. These tori are parametrized by the corresponding values of the integrals of motion $\left(E_{0}, l_{z}, A_{z}\right)$. For fixed energy $E_{0}<0$, the $z$ projection of the angular momentum takes values from the interval $-1 / \sqrt{-2 E_{0}}$ to $1 / \sqrt{-2 E_{0}}$, while for given $E_{0}$ and $l_{z}$, the $z$ projection of the Runge-Lentz vector takes the values from the interval $-1+\left|l_{z}\right| \sqrt{-2 E_{0}}$ to $1-\left|l_{z}\right| \sqrt{-2 E_{0}}$ i.e., there is a one-to-one correspondence between the tori that foliate the energy shell $E_{0}$ and the points that belong to the open subset (the interior of the parallelogram defined above) of the $l_{z}-A_{z}$ plane; see Fig. 8. Note that the points that belong to the closure of the parallelogram are all critical points of the corresponding energy-momentum map. Therefore, the initial state can be described with a distribution function defined on the set of all possible pairs $\left(l_{z}, A_{z}\right)$ for the given value of the initial energy $E_{0}$. It is not hard to show that the distribution function corresponding to the microcanonical ensemble Eq. (16) is given by

$$
d w\left(l_{z}, A_{z}\right)=\left(-E_{0} / 2\right)^{1 / 2} d l_{z} d A_{z},
$$

i.e., the probability distribution of the phase-space points of the microcanonical ensemble Eq. (16) corresponds to the uniform distribution of the corresponding tori (that foliate the energy shell) when parametrized by $l_{z}$ and $A_{z}$.

Given the values of the integrals of motion $\left(E_{0}, l_{z}, A_{z}\right)$, let $T_{E_{0}, l_{z}, A_{z}}^{3}$ denote the initial invariant torus. The corresponding action variables are determined by the formulas

$$
\begin{aligned}
& S_{1}=\frac{1+A_{z}}{2\left(-2 E_{0}\right)^{1 / 2}}-\frac{\left|l_{z}\right|}{2}, \\
& S_{2}=\frac{1-A_{z}}{2\left(-2 E_{0}\right)^{1 / 2}}-\frac{\left|l_{z}\right|}{2}, \\
& S_{3}=l_{z} .
\end{aligned}
$$
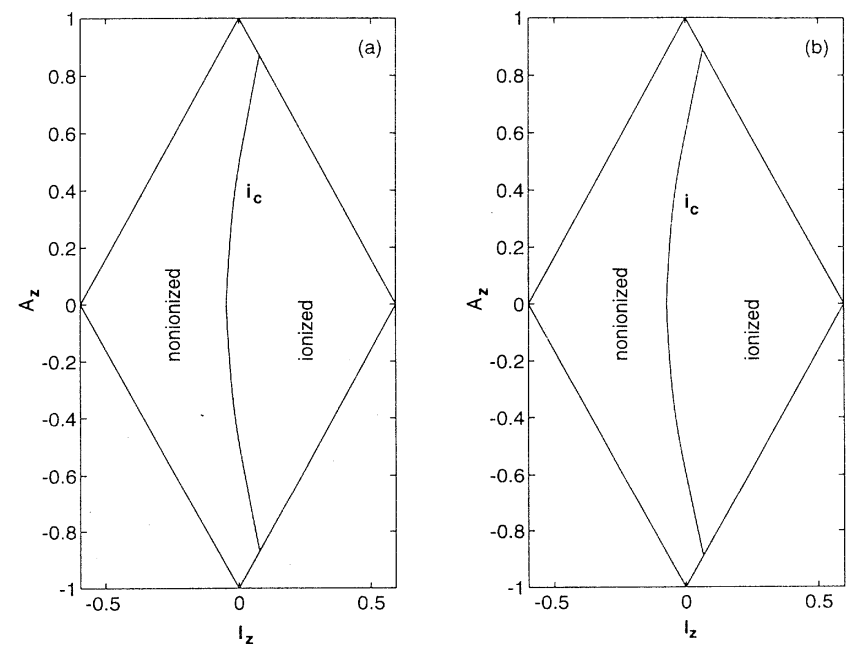

FIG. 8. (a) Set of all possible values of $\left(l_{z}, A_{z}\right)$ corresponding to tori that foliate the energy shell of the (unperturbed) hydrogen atom with $E_{0}=-1.410$. The line $i_{c}$ is the solution of the Eq. (21) for $\omega=1$ and $f_{*}=1.35$. The probability of ionization (proportional to the "ionized" area) is $w=0.490$. (b) Same as (a) but for $E_{0}=-1.397$. Then $w=0.522$. All quantities are given in atomic units. 
Note that the energy in the rotating frame is $E_{\text {cir }}(f=0)=E_{0}-\omega l_{z}$. Suppose now that the circular field is adiabatically switched on. Due to the near integrability of the system $I$, i.e., of the Hamiltonian function $H_{\text {cir }}$, Eq. (1), described in the previous sections, the majority of the tori will survive perturbation even for large values of $f$. As $f$ adiabatically increases the initial torus $T_{E_{0}, l_{z}, A_{z}}^{3}$ deforms and forms a one-parameter family $T_{E_{0}, l_{z}, A_{z}}^{3}(f)$, while preserving the values of its actions, Eqs. (18)-(20), since they are adiabatic invariants [8]. Eventually, for some value of the field $f_{*}=f_{*}\left(E_{0}, l_{z}, A_{z}\right)$ the torus "breaks up" and the corresponding trajectories become unbounded; this is interpreted as the ionization of the hydrogen atom. Now we shall us the correspondence between the tori of systems I and II (see Sec. III): given the values of the parameters $f$ and $\omega$ to any invariant torus of system I corresponds the nearby-lying invariant torus of system II, which has the same values of the actions. Therefore, to each initial invariant torus of the unperturbed hydrogen atom, i.e., to each corresponding triplet $\left(E_{0}, l_{z}, A_{z}\right)$, we can associate a curve $\left(E(f), C_{1}(f), C_{2}(f)\right.$ ) (in the set $\left.Q_{1}\right)$ defined with the condition that the invariant tori (from the family $w_{1}$ ) that correspond to the points from that curve have the same values for action variables as the initial torus, i.e., that $\left(S_{1}, S_{2}, S_{3}\right)=\chi_{1}\left(E(f), C_{1}(f), C_{2}(f)\right)$. The corresponding one-parameter family of invariant tori $T_{E(f), C_{1}(f), C_{2}(f)}^{3}$ is obtained by the continuous deformation of the torus $T_{E(0), C_{1}(0), C_{2}(0)}^{3}$ when the parameter $f$ in the Hamiltonian function $H$, Eq. (2), is adiabatically increased. The parameter $f$ can be increased until it reaches the point of bifurcation $f_{c}=f_{c}\left(S_{1}, S_{2}, S_{3}\right)=f_{c}\left(E_{0}, l_{z}, A_{z}\right)$ for which the curve $\left(E(f), C_{1}(f), C_{2}(f)\right)$ intersects the critical surface $\epsilon_{1}$ (i.e., for which the corresponding torus meets another invariant torus, of the family $w_{2}$; see Sec. II B). Suppose now that for each $f \in\left[0, f_{c}\right)$ the corresponding torus $T_{E(f), C_{1}(f), C_{2}(f)}^{3}$ belongs to $w_{\text {cir }}$, i.e., it corresponds to the invariant torus of system I; see Sec. III. In that case the field intensity $f_{*}$ for which the invariant torus of system I breaks up can be approximated with the bifurcation point $f_{c}$ of the corresponding invariant torus of system II, i.e., we can write

$$
f_{*}=f_{c}\left(S_{1}, S_{2}, S_{3}\right)=f_{c}\left(E_{0}, l_{z}, A_{z}\right) .
$$

In summary, the method we have used to calculate the field intensity $f_{*}$ for which the given initial invariant torus of the unperturbed hydrogen atom breaks up is the following. First, from the given values of the integrals of the motion $\left(E_{0}, l_{z}, A_{z}\right)$ we calculate, using Eqs. (18)-(20), the corresponding action variables $\left(S_{1}, S_{2}, S_{3}\right)$. Then, for each $f$ we calculate the inverse of the function $\chi_{1}$, Eq. (15), at the point $\left(S_{1}, S_{2}, S_{3}\right)$, thus locating the continuous curve $\left(E(f), C_{1}(f), C_{2}(f)\right)$ in the set $Q_{1}$ together with the point $f_{c}$ at which the curve intersects the critical surface $\epsilon_{1}$. If all points $\left(E(f), C_{1}(f), C_{2}(f)\right)$ belong to the set $Q_{\text {cir }} \subset Q_{1}$, we identify the bifurcation point $f_{c}$ with $f_{*}$.

Let us now fix the field intensity $f_{*}$ and the initial en- ergy $E_{0}$. Then the solution of Eq. (21) defines a curve in the $l_{z}-A_{z}$ plane that may have a nonempty intersection (we denote it with $i_{c}$ ) with the set of all possible pairs $\left(l_{z}, A_{z}\right)$ for the given energy $E_{0}$. Examples are given in Figs. 8(a) and 8(b) for $\omega=1, f_{*}=1.35$, and $E_{0}=-1.410,-1.397$. Clearly the points that belong to the curve $i_{c}$ correspond to initial invariant tori that break up when the field intensity reaches the given value $f_{*}$. The curve $i_{c}$ divides the set of pairs $\left(l_{z}, A_{z}\right)$ into two subsets, one of which, as indicated in Figs. 8(a) and 8(b), corresponds to initial tori that have already been "broken" (or "ionized") for the field intensity less than $f_{*}$, while the other subset corresponds to initial tori that will be broken for greater field intensities.

As follows from Eq. (17), the volume of this ionized subset $p_{f_{*}}\left(E_{0}\right)$ is proportional to the ionization probability $w_{f_{*}}\left(E_{0}\right)$ of the initial (hydrogenic) state with the energy $E_{0}$ for the field intensity $f_{*}$, i.e.,

$$
w_{f_{*}}\left(E_{0}\right)=\left(-E_{0} / 2\right)^{1 / 2} p_{f_{*}}\left(E_{0}\right) \text {. }
$$

To be consistent with the experiment we define the ionization threshold field (see Ref. [3]) as the field intensity for which half of the initial ensemble is ionized. That is, given the energy $E_{0}$ of the initial hydrogenic state, the ionization threshold field is defined as the intensity of the field $f_{\text {th }}$ for which

$$
w_{f_{\mathrm{th}}}\left(E_{0}\right)=\frac{1}{2} \text {. }
$$

Following the method presented in this section we have been able to calculate (classical) ionization threshold fields for initially highly excited states of the hydrogen atom. The results are given in the Table I. In Fig. 9 we give the ionization thresholds fields as a function of the initial principal quantum number of the hydrogen atom $n_{0}=1 / \sqrt{-2 E_{0}}$ for $\omega=8.5 \mathrm{GHz}$ and $22<n_{0}<55$.

As observed in the experiment [3] (on the Rydberg states of sodium), the threshold fields for ionization of the hydrogen atom in circularly polarized microcanonical fields practically obey the scaling law $f_{\text {th }}=1 / c n_{0}^{4}$ characteristic for the classical static-field case. We found that for the hydrogen atom, $c$ is almost a constant, $c \approx 6$. The change of the parameter $c(5.784-5.857)$ is not observable in the scales of Fig. 9. These results are similar to those for the case of the restricted motion in the plane of polarization [5], where the same threshold law was obtained, but with the parameter $c$ taking values from the interval $5.973-6.100$.

TABLE I. Calculated ionization threshold fields for initially highly excited states of the hydrogen atom.

\begin{tabular}{crc}
\hline \hline$n_{0}=1 / \sqrt{-2 E_{0}}$ & $f_{\text {th }}(\mathrm{V} / \mathrm{cm})$ & $c=1 / f_{\text {th }} n_{0}^{4}$ (a.u.) \\
\hline 23.838 & 2752.097 & 5.784 \\
30.083 & 1084.784 & 5.785 \\
34.178 & 650.870 & 5.788 \\
39.133 & 378.445 & 5.791 \\
46.258 & 193.092 & 5.814 \\
54.755 & 97.631 & 5.857 \\
\hline \hline
\end{tabular}




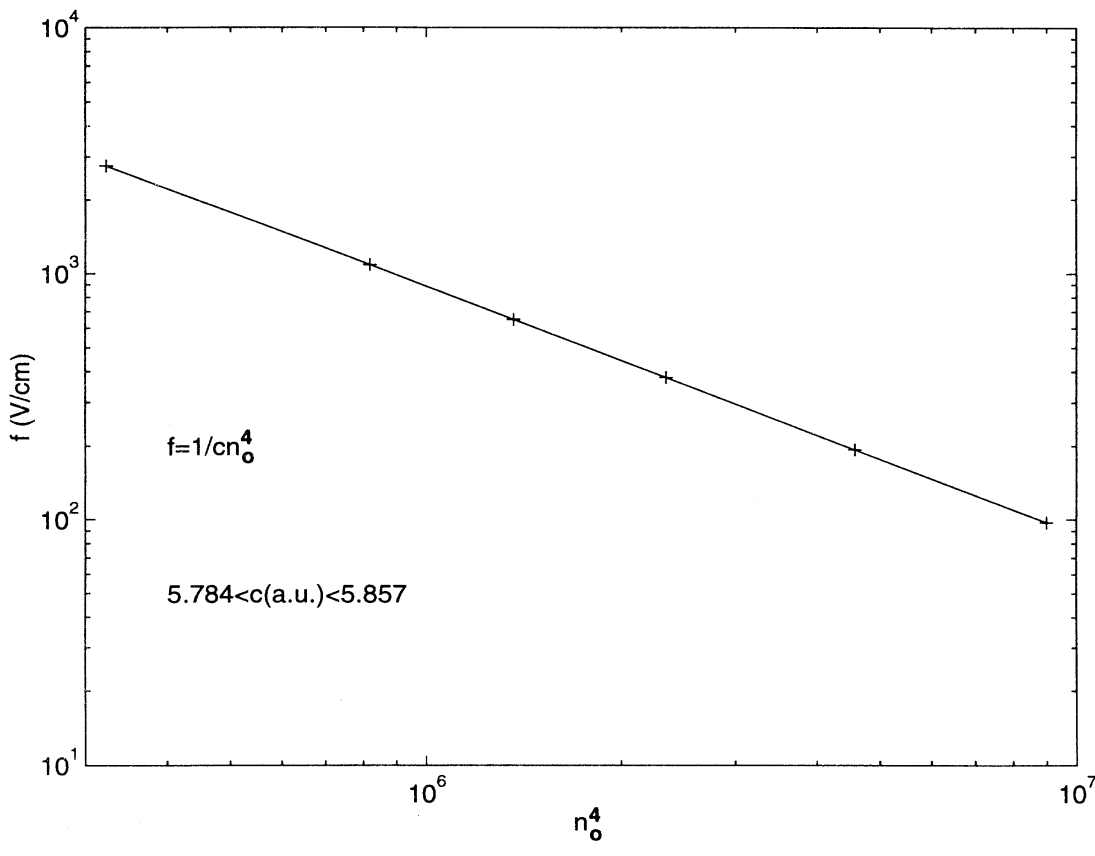

FIG. 9. Ionization threshold field as a function of the principal quantum number $n_{0}$ corresponding to the initial state of the hydrogen atom. The frequency of the microwave field is $\omega=8.5 \mathrm{GHz}$.
For comparison we have also calculated [12] the ionization field thresholds for the hydrogen atom in the case of the static field where the scaling law is exact. The result $c_{\text {st }}=5.783$ is very close to the values obtained in the case of the circularly polarized microwave field; see Table I.

In conclusion, we comment on the relation between our result and the experiments. In the experiment on the ionization of highly excited states of the sodium atom in circularly polarized microwave fields [3], the threshold law $f_{\text {th }} / 16 n_{0}^{4}$ obtained was close to the static-field case. These ionization threshold fields are therefore considerably higher than those in the case of the linearly polarized field where $f_{\text {th }} \approx 1 / 3 n_{0}^{5}$. On the other hand, the threshold field for the hydrogen atom in linearly polarized field is $1 / 9 n_{0}^{4}$ [3]. Hence it was expected that in the case of the hydrogen atom and the circular field the threshold fields should be above $1 / 9 n_{0}^{4}$ and close to the static-field values. Our result is in accord with such expectations; moreover, it is in excellent agreement with preliminary experimental results [6].

\section{ACKNOWLEDGMENTS}

M.J.R. acknowledges the support of the Kansas Institute for Theoretical and Computational Science through the $\mathrm{K}^{*} / \mathrm{STAR} / \mathrm{NSF}$ program. S.I.C. is supported by the National Science Foundation under the Contract No. PHY-9207277.

\section{APPENDIX: ANALYTICAL EXPRESSIONS FOR THE CRITICAL POINTS}

All equations in the Appendix are formulated in scaled variables. Let us fix the scaled field intensity $f_{s}$. Consider first the intersection of the set $Q_{c}$ with the plane $C_{2 s}=0$. When $f_{s}>\left(\frac{4}{9}\right)^{2 / 3}$ the curves $l_{1}, l_{2}, l_{3}, \sigma_{1}, \sigma_{2}$ and the segment $F_{1} A$ of the curve $\epsilon_{2}$ in Fig. 1(a) are all parametrized by the same pair of equations

$$
\begin{aligned}
& E_{s}=-2 \theta / 3+9 f_{s}^{2} / 8-1 /\left(2 \theta^{2}\right), \\
& C_{1 s}=-2 \theta^{2} / 9-2 /(3 \theta)
\end{aligned}
$$

and each has its own range of the parameter $\theta$

$$
-\infty<\theta_{l_{1}} \leq-\frac{2}{3 f_{s}}<\theta_{\sigma_{2}}<0<\theta_{\sigma_{1}}<\theta_{F_{1}}<\theta_{\epsilon_{2}}<\frac{2}{3 f_{s}}<\theta_{l_{2}}<\left(\frac{3}{2}\right)^{1 / 3}<\theta_{l_{3}}<\theta_{F_{2}},
$$

where

$$
\begin{aligned}
\theta_{F_{1,2}}= & \mp 3 f_{s} r_{ \pm} / 4-r_{ \pm}^{2} / 3 \\
& +\left[\left(\mp 3 f_{s} r_{ \pm} / 4-r_{ \pm}^{2} / 3\right)^{2}+r_{ \pm}\right]^{1 / 2}
\end{aligned}
$$

and $r_{ \pm}$is the positive root of the cubic equation

$$
8 r^{3} / 9 \pm f_{s} r^{2}-1=0 \text {. }
$$

When $f_{s} \leq\left(\frac{4}{9}\right)^{2 / 3}$ the curves $l_{2}$ and $l_{3}$ do not exist, the triangle $A F_{2} B$ is reduced to a point $F_{2}$, and for the range of $\theta$ that corresponds to $\epsilon_{2}$ we have

$$
\theta_{F_{1}}<\theta_{\epsilon_{2}}<\theta_{F_{2}}
$$

The curve $\epsilon_{1}$ in the Fig. 1(a) is determined with 


$$
C_{1 s}=-\frac{E_{s}^{2}}{2}+f_{s}, \quad\left(-3-f_{s} r_{+}^{2}\right) /\left(2 r_{+}\right)<E_{s}<\frac{4}{9 f_{s}},
$$

while for the segment of $\epsilon_{2}$, on the right from the point $A$ (or $F_{2}$ ), we have

$$
\begin{aligned}
C_{1 s}= & -\frac{E_{s}^{2}}{2}-f_{s} \\
& -\frac{4}{9 f_{s}}<E_{s}<\infty \text { if } f_{s}>\left(\frac{4}{9}\right)^{2 / 3},
\end{aligned}
$$

$$
\left(-3+f_{s} r_{-}^{2}\right) /\left(2 r_{-}\right)<E_{s}<\infty \text { if } f_{s}<\left(\frac{4}{9}\right)^{2 / 3} .
$$

Now we shall formulate equations that define critical points for which $C_{2 s} \neq 0$. Let $X \subset \mathbb{R}^{3}$ be the set of all points $(\rho, \phi, z)$ that satisfy the equation

$$
\begin{aligned}
a_{4}(\rho, \phi) z^{4}+a_{3}(\rho, \phi) z^{3}+a_{2}(\rho, \phi) z^{2} & \\
& +a_{1}(\rho, \phi) z+a_{0}(\rho, \phi)=0,
\end{aligned}
$$

where

$a_{4}(\rho, \phi)=\rho^{2}\left(1+4 \cos ^{2} \phi+3 \cos ^{4} \phi\right)$,

$a_{3}(\rho, \phi)=-\frac{4}{3} \rho^{3} \sin \phi \cos \phi\left(1+\cos ^{2} \phi\right)+3 f_{s} \rho^{2} \sin \phi\left(1+\frac{13}{2} \cos ^{2} \phi+\frac{13}{2} \cos ^{4} \phi\right)$,

$a_{2}(\rho, \phi)=-2 f_{s} \rho^{3} \cos \phi\left(3+\cos ^{2} \phi-4 \cos ^{4} \phi\right)+\frac{9}{4} f_{s}^{2} \rho^{2}\left(1+13 \cos ^{2} \phi+7 \cos ^{4} \phi-21 \cos ^{6} \phi\right)-2 \rho\left(1+\cos ^{2} \phi-2 \cos ^{4} \phi\right)$,

$a_{1}(\rho, \phi)=\frac{2}{3} f_{s} \rho^{4} \sin ^{3} \phi\left(1+\cos ^{2} \phi\right)-3 f_{s}^{2} \rho^{3} \sin \phi \cos \phi\left(2+3 \cos ^{2} \phi-5 \cos ^{4} \phi\right)$

$$
+\frac{9}{4} \rho^{2} \sin \phi \cos \phi\left[\frac{16}{27} \sin ^{2} \phi+f_{s}^{3}\left(\frac{15}{2} \cos \phi+15 \cos ^{3} \phi-\frac{45}{2} \cos ^{5} \phi\right)\right]-3 f_{s} \rho \sin \phi\left(1+3 \cos ^{2} \phi-4 \cos ^{4} \phi\right),
$$

$a_{0}(\rho, \phi)=f_{s}^{2} \rho^{4} \sin ^{4} \phi \cos ^{2} \phi-9 f_{s}^{3} \rho^{3} \cos ^{3} \phi \sin ^{4} \phi+2 f_{s} \rho^{2} \sin ^{4} \phi \cos \phi\left(1+\frac{81}{8} f_{s}^{3} \cos ^{3} \phi\right)-9 f_{s}^{2} \rho \sin ^{4} \phi \cos ^{2} \phi+\sin ^{4} \phi$,

and for which

$$
\sin \phi \neq 0, \quad 6 \rho^{2} z \cos \phi-4 \rho^{3} \sin \phi+9 f_{s} \rho^{2} \sin \phi \cos \phi \neq 0, \quad p(\rho, \phi, z)=\rho^{2} z^{4}+\frac{3}{2} f_{s} \rho^{2} z^{3} \sin \phi>0 .
$$

holds. Then, the map defined with the equations

$$
\begin{aligned}
E_{s}(\rho, \phi, z)= & \frac{1+\cos ^{2} \phi}{\sin ^{2} \phi} z^{2}+\left[f_{s} \frac{9+15 \cos ^{2} \phi}{4 \sin \phi}-\frac{8}{3} \rho \cot \phi\right] z+\frac{4}{9} \rho^{2}-3 f_{s} \rho \cos \phi+\frac{27}{8} f_{s}^{2} \cos ^{2} \phi+\frac{9}{8} f_{s}^{2}-\frac{1}{\rho}, \\
C_{1 s}(\rho, \phi, z)= & \frac{8 \rho \cos \phi\left(1+\cos ^{2} \phi\right)}{3 \sin ^{3} \phi} z^{3} \\
& +\left[-4 \rho^{2} \frac{1+9 \cos ^{2} \phi}{9 \sin ^{2} \phi}+f_{s} \rho \cos \phi \frac{7+13 \cos ^{2} \phi}{\sin ^{2} \phi}\right] z^{2} \\
& +\left[\frac{32}{27} \rho^{3} \cot \phi-f_{s} \rho^{2} \frac{3+29 \cos ^{2} \phi}{3 \sin \phi}+\frac{1}{2} f_{s}^{2} \rho \cot \phi\left(9+39 \cos ^{2} \phi\right)-\frac{8}{3} \cot \phi\right) z \\
& -\frac{8}{81} \rho^{4}+\frac{4}{3} f_{s} \rho^{3} \cos \phi-6 f_{s}^{2} \rho^{2} \cos ^{2} \phi+\left(\frac{4}{9}+9 f_{s}^{3} \cos ^{3} \phi\right) \rho-3 f_{s} \cos \phi, \\
C_{2 s}(\rho, \phi, z)= & \pm \sqrt{p(\rho, \phi, z)}
\end{aligned}
$$

maps the set $X$ onto the set of all critical points that belong to surfaces $\sigma_{1}, \sigma_{2}, \epsilon_{1}$, and $\epsilon_{2}$ and for which $C_{2 s} \neq 0$.

Finally, we have to give the formula for the critical curve $l_{4}$. We first define the set $Y \subset \mathbb{R}^{2}$ as the set of all points $(\rho, \phi)$ that satisfy the equation

$$
\left(\frac{9}{4}-\rho^{3}\right) \cos ^{2} \phi+\frac{9}{4} f_{s} \rho^{2} \cos \phi-\rho^{3}=0
$$

and inequalities

$$
p(\rho, \phi)=\rho\left(\frac{4}{9} \rho-f_{s} \cos \phi\right) \tan ^{2} \phi>0, \quad \cos \phi>0, \quad 0<\rho<\rho_{\max }
$$

where

$$
\begin{aligned}
& \rho_{\max }=\left(\frac{9}{4}\right)^{1 / 3} \text { if } f_{s} \geq\left(\frac{4}{9}\right)^{2 / 3}, \\
& \rho_{\max }=r_{-} \text {if } f_{s}<\left(\frac{4}{9}\right)^{2 / 3} .
\end{aligned}
$$

Then the map defined with the equations

$$
E_{s}(\rho, \phi)=\frac{4}{9} \frac{\rho^{2}}{\cos ^{2} \phi}-\frac{8}{9} \rho^{2}-\frac{1}{2} f_{s} \frac{\rho}{\cos \phi}+\frac{3}{2} f_{s} \rho \cos \phi-\frac{1}{\rho},
$$



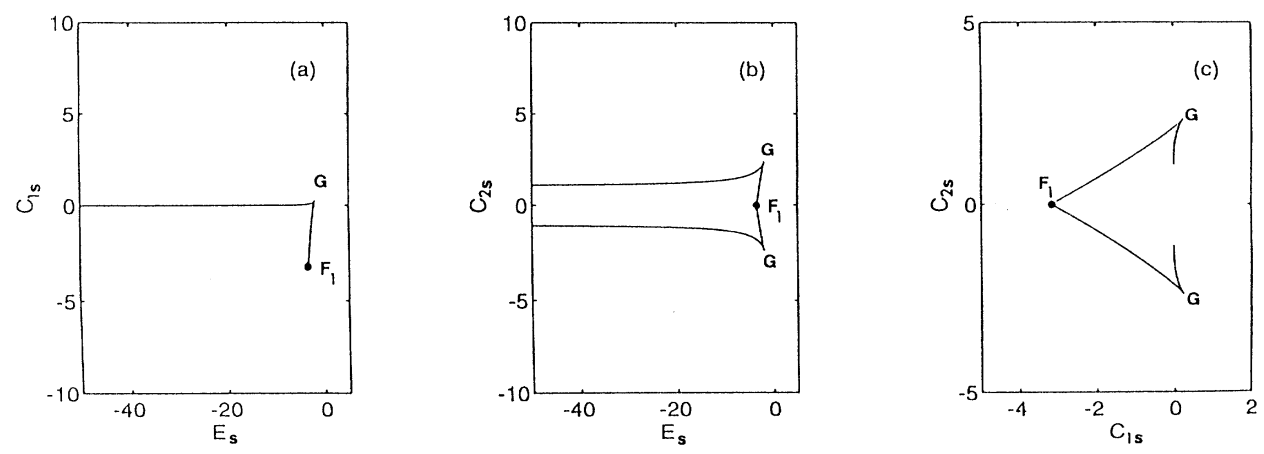

FIG. 10. Plot of the common subset of the critical surfaces $\sigma_{1}$ and $\epsilon_{2}$ for the case $f_{s}=2.67$. (a) Projection onto the $E_{s}-C_{1 s}$ plane. (b) Projection onto the $E_{s}-C_{2 s}$ plane. (c) Projection onto the $C_{1 s}-C_{2 s}$ plane. All quantities are given in atomic units.

$$
\begin{aligned}
& C_{1 s}(\rho, \phi)=-\frac{3}{2} f_{s}^{2} \rho^{2} \cos ^{2} \phi+f_{s}\left(1+\frac{14}{9} \rho^{3}\right) \cos \phi+\frac{3}{2} f_{s}^{2} \rho^{2}-\frac{4}{3} \rho-\frac{8}{27} \rho^{4}-2 f_{s} \frac{\rho^{3}}{\cos \phi}+\frac{16}{27} \frac{\rho^{4}}{\cos ^{2} \phi}, \\
& C_{2 s}(\rho, \phi)= \pm \frac{3}{2} \sqrt{p(\rho, \phi)^{3}} \cot \phi
\end{aligned}
$$

maps the set $Y$ onto the critical curve $l_{4}$.

It appears that the common subset of the critical surfaces $\sigma_{1}$ and $\epsilon_{2}$ is a nonsmooth but continuous curve; the points $F_{1}, g_{1}$, and $g_{2}$ in Figs. 1(a)-1(c) belong to that curve. This curve is determined by the same equations (given above) as the critical curve $l_{4}$, except that for the domain of points $(\rho, \phi)$ one has

$\cos \phi<0, \quad 0<\rho<r_{+}$.

In Figs. 10(a) -10 (c) we give the plots of that curve for the case $f_{s}=2.67$. We see that the point $G$ in these figures corresponds to maximal value $C_{2 G}=C_{2 G}\left(f_{s}\right)$ for which the regular set $Q_{1}$ has a nonempty intersection with the $C_{2}$ planes; see Sec. II A and Figs. 1(a)-1(c).

[1] A number of microwave ionization experiments on Rydberg atoms in linearly polarized fields have been performed. See, for example, J. E. Bayfield and L. A. Pinnaduwage, Phys. Rev. Lett. 54, 313 (1985); K. A. H. van Leeuwen, G. V. Oppen, S. Renwick, J. B. Bowlin, P. M. Koch, R. V. Jensen, O. Raht, D. Richards, and J. G. Lepold, ibid. 55, 2231 (1985); J. E. Bayfield and D. W. Sokol, ibid. 61, 2007 (1988).

[2] For a recent account of theoretical work on microwavedriven multiphoton ionization of Rydberg $\mathbf{H}$ atoms in linearly polarized fields, see G. Casati, B. V. Chirikov, D. L. Shepelyansky, and I. Guarnieri, Phys. Rep. 154, 77 (1987), and references therein, R. Jensen ibid. 201, 1 (1991).

[3] P. Fu, T. Scholz, J. M. Hettema, and T. F. Gallagher, Phys. Rev. Lett. 64, 511 (1990).

[4] For classical studies see P. Kappertz and M. Nauenberg, Phys. Rev. A 47, 4749 (1993); J. A. Griffiths and D. Farrelly, ibid. 45, R2678 (1992); K. Rzazewski and B. Piraux, ibid. 47, 1612 (1993). For a quantum approach see J. Zakrzewski, D. Delande, J.-C. Gay, and K. Rzazewski, ibid. 47, 2468 (1993).

[5] M. J. Raković and Shih-I Chu, Phys. Rev. A 50, 5077 (1994).

[6] M. W. Bellermann, P. M. Koch, D. R. Mariani, and D. Richards, Microwave Ionization of $\mathbf{H}$ Atoms for Circular (CP), Elliptical (EP) and Linear (LP) Polarization, 1994, Joint Meeting of the American Physical Society and American Association of Physics Teachers [Bull. Am. Phys. Soc. 39, 1120 (1994)].

[7] M. J. Raković and Shih-I Chu, Physica D 81, 271 (1995).

[8] V. I. Arnol'd, Mathematical Methods of Classical Mechanics (Springer, Berlin, 1978).

[9] M. J. Raković and Shih-I Chu (unpublished).

[10] M. Antonowicz, J. Phys. A 14, 1099 (1981).

[11] R. Cushman and J. J. Dustermaat, Bull. Am. Math. Soc. 19, 475 (1988).

[12] M. J. Raković (unpublished). 